\title{
FINITE ELEMENT FORMULATION AND FREE VIBRATION ANALYSES OF ROTATING, FUNCTIONALLY GRADED BLADES
}

\author{
Elif D. Karahan, Özge ÖZdemiR \\ Istanbul Technical University, Faculty of Aeronautics and Astronautics, Maslak, Istanbul, Turkey \\ e-mail: ozdemirozg@itu.edu.tr
}

\begin{abstract}
In this study, free vibration characteristics of functionally graded (FG) blades whose material properties change through the blade thickness are inspected. Finite Element Method (FEM) is used to create blade models and to calculate natural frequencies. The blade formulations are derived for both Euler-Bernoulli and Timoshenko beams to inspect the effect of different parameters on vibration characteristics. For each beam, stiffness and mass matrices are derived from energy expressions. In the solution part, effects of several parameters, i.e. rotational speed, material properties, power law index parameter, different boundary conditions and slenderness ratio are investigated. The calculated results are compared with those in open literature and a very good agreement between them is confirmed, which reveals the correctness and accuracy of the finite element formulation developed in this study. Formulations are carried out in great detail and additionally, the results are displayed in several figures and tables, which can be a significant source of information for authors working in this area.
\end{abstract}

Keywords: helicopter blade, functionally graded material, finite element method

\section{Introduction}

In industry and in lots of engineering applications, rotating components, turbines, helicopter blades, rotors, etc. have a wide area of utility. Design, material properties and dynamic properties of these structures and their components have significant effects on system efficiency. Helicopters have different vibration problems since they consist of many components that are connected to each other and since they have a complex rotating system. Therefore, frequencies and mode shapes are used to determine dynamic properties of such structures.

The aim of this study is to develop a computer code by using the finite element method for vibration analysis of a FG helicopter rotor blade whose material properties change in the thickness direction. Functionally graded materials (FGMs) that have gained widespread application are used to increase functional performance enhanced by the desired gradient of the material properties. This variation provides continuous stress distribution in FG structures, whereas discontinuous stress distribution appears in another type of advanced materials, i.e. laminated composites. Material properties of the beam such as elastic modulus, shear modulus, Poisson's ratio, material density are assumed to change continuously through the thickness direction as a function of volume fraction and according to a simple power law. The concept of FGMs originated from a group of material scientists in Japan as means of preparing thermal barrier materials (Loy et al., 1999), and shortly began to take place in engineering applications at a near future. FGM resulting from the development of composite material technology is a new generation material that enables advanced engineering applications by reflecting mechanical, physical and chemical properties of the materials it is made of. Nowadays, material properties and composition of FGM, whose production areas and application fields are increasing day by day with the development of additive manufacturing technology and powder metallurgy, are 
changing throughout the structure. The material change takes place in the form of a gradient connected to a function. Due to these properties, functional graded materials are used and applied in many different sectors such as aerospace, automotive and medical. Several research papers provide a good introduction and further references on the subject (Alshorbagy et al., 2011; Giunta et al., 2011; Huang and Li, 2010; Lai et al., 2012; Loja et al., 2012; Thai and Vo, 2012; Wattanasakulpong et al., 2012).

In this study, FG blades whose material distribution changes in the thickness direction are modeled, and vibration analyses are performed. In these studies, beam models with different boundary conditions and material properties are investigated. For developing mathematical models and for the solution, the finite element method (FEM) is used. The blade formulations are derived for both Euler-Bernoulli and Timoshenko beam theories to inspect the effect of different parameters on vibration characteristics. For each beam theory, element stiffness and mass matrices are derived from energy expressions. In the solution part, effects of several parameters, i.e. rotational speed, material properties, power law index parameter, different boundary conditions, rotary inertia, shear deformation and slenderness ratio are investigated. The calculated results are compared with those in open literature and a very good agreement between them is found, which reveals the correctness and accuracy of the finite element formulation developed in this study.

\section{Functionally graded beam model}

In this study, energy expressions of a rotating beam which has different material properties in different compositions changing through blade thickness, see Fig. 1., are used.

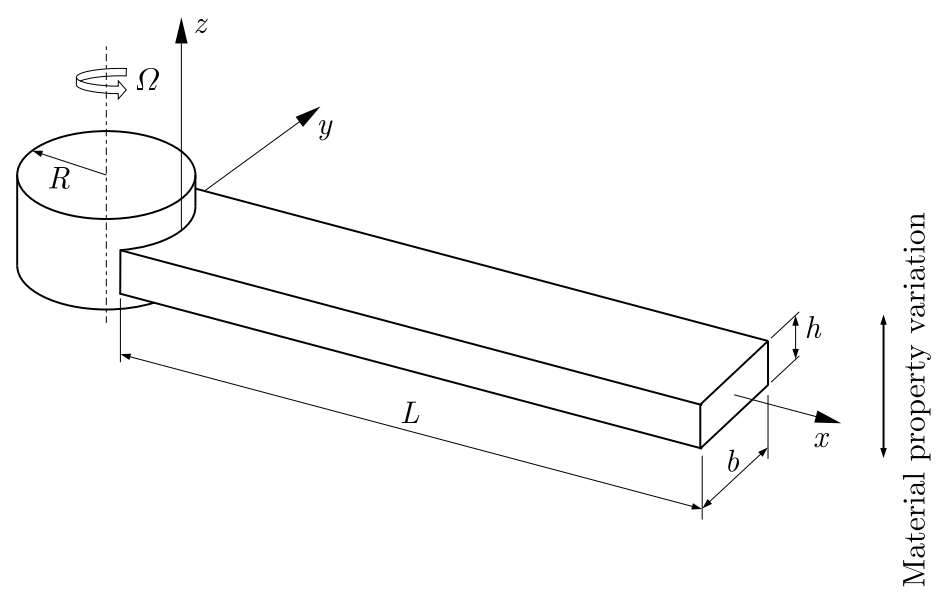

Fig. 1. Rotating, FG beam model

Here, a rotating FG beam of length $L$ is fixed to a rigid hub with radius $R$. The beam height is $h$ and width is $b$, and the $x y z$ axes represent a global orthogonal coordinate system with its origin at the root of the beam. The beam is assumed to be rotating in the counter-clockwise direction at a constant angular velocity $\Omega$.

Material properties of the beam, i.e. modulus of elasticity $E$, shear modulus $G$, Poisson's ratio $\nu$ and material density $\rho$ are assumed to vary continuously in the thickness direction $z$ as a function of the volume fraction, and the properties of the constituent materials according to a simple power law. According to the rule of mixture, the effective material property $P(z)$ is

$$
P(z)=P_{t} V_{t}+P_{b} V_{b}
$$


where $P_{t}$ and $P_{b}$ are material properties at the top and bottom surfaces of the beam while $V_{t}$ and $V_{b}$ are corresponding volume fractions. The relation between the volume fractions is

$$
V_{t}+V_{b}=1
$$

The volume fraction of the top constituent of the beam $V_{t}$ is assumed to be given by

$$
V_{t}=\left(\frac{z}{h}+\frac{1}{2}\right)^{n} \quad n \geqslant 0
$$

Here $n$ is a non-negative power law index parameter that dictates the material variation profile through the beam thickness.

Considering Eqs. (2.1)-(2.3), the effective material properties can be rewritten as follows

$$
P(z)=\left(P_{t}-P_{b}\right)\left(\frac{z}{h}+\frac{1}{2}\right)^{n}+P_{b}
$$

It is evident from Eq. (2.4) that when $z=h / 2, E=E_{t}, \nu=\nu_{t}, G=G_{t}, \rho=\rho_{t}$ and when $z=-h / 2, E=E_{b}, \nu=\nu_{b}, G=G_{b}$ and $\rho=\rho_{b}$.

\section{Energy expressions}

In this Section, the potential and kinetic energy expressions are given both for a rotating Euler-Bernoulli beam and a rotating Timoshenko beam. The steps of derivation can be found in some well-known papers in literature (Han et al., 1999; Latalski et al., 2017) in great detail by using several explanatory figures and tables.

The cross-sectional and the longitudinal views of the Euler-Bernoulli beam that undergoes axial displacement $u_{0}$ and transverse displacement $w$ are given in Figs. $2 \mathrm{a}$ and $2 \mathrm{~b}$, respectively. Here, the reference point is chosen and represented by $P_{0}$ before deformation and by $P$ after deformation.

(a)

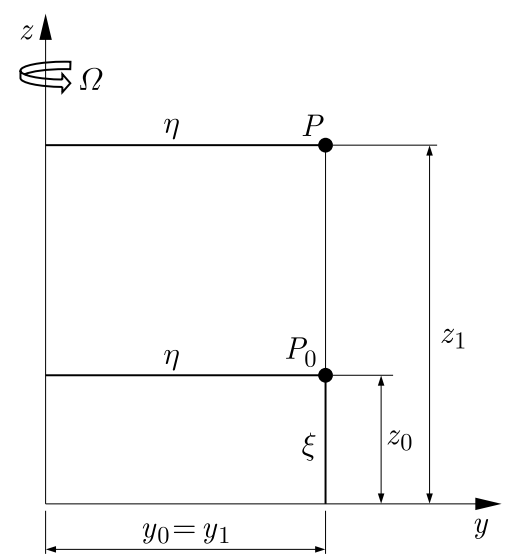

(b)

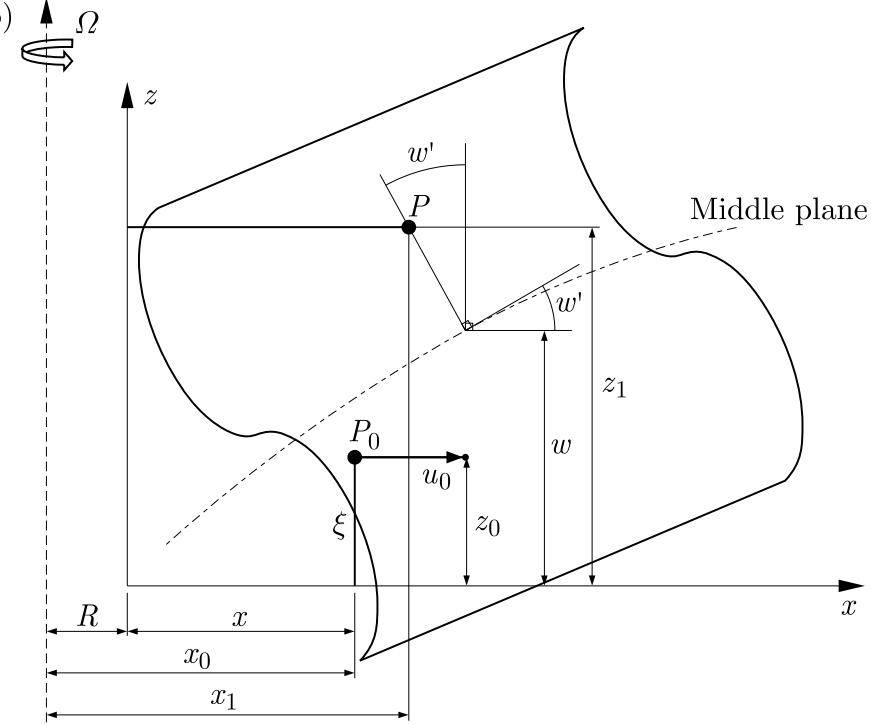

Fig. 2. (a) Cross-sectional view, (b) longitudinal view of a rotating Euler-Bernoulli beam

In Fig. 2, $y$ and $z$ are the global coordinates while $\eta$ and $\xi$ are the corresponding local coordinates. Here, $\eta$ is the offset of the reference point from the $z$-axis, $\xi$ is the offset of the reference point from the middle plane, $x$ is the offset of the reference point from the $z$-axis. 
(a)

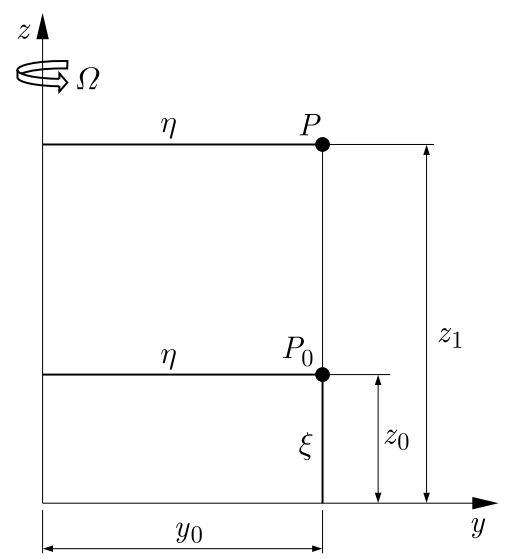

(b)

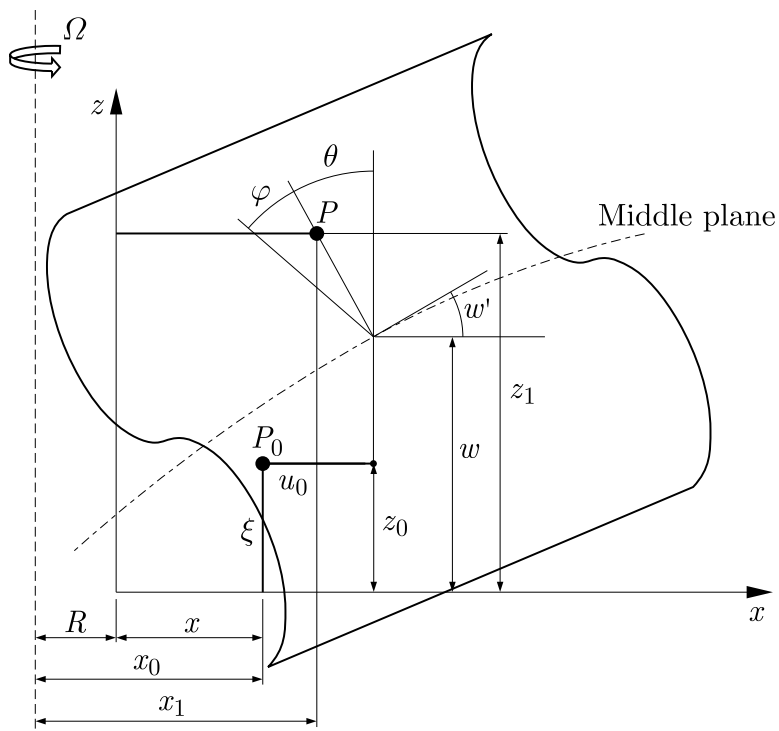

Fig. 3. (a) Cross-sectional view, (b) longitudinal view of a rotating Timoshenko beam

The cross-sectional and the longitudinal views of the Timoshenko beam that undergoes axial displacement $u$ and transverse displacement, $w$ are given in Figs. 3a and 3b, respectively.

Here, $\theta$ is rotation due to bending, and $\varphi$ is the shear angle.

The potential energy expressions of the rotating Euler-Bernoulli and Timoshenko beam are given in Eqs. $(3.1)_{1}$ and $(3.1)_{2}$, respectively

$$
\begin{aligned}
& U_{\text {Euler }}=\frac{1}{2} \int_{0}^{L}\left[\bar{D}_{11}\left(\theta^{\prime}\right)^{2}+F_{C F}(x)\left(w^{\prime}\right)^{2}\right] d x \\
& U_{\text {Timoshenko }}=\frac{1}{2} \int_{0}^{L}\left[\bar{A}_{11}\left(u_{0}^{\prime}\right)^{2}+\bar{B}_{11} u_{0}^{\prime} \theta^{\prime}+\bar{D}_{11}\left(\theta^{\prime}\right)^{2}+\bar{A}_{55}\left(w^{\prime}-\theta\right)\right] d x
\end{aligned}
$$

Here, the stiffness coefficients are

$$
\left[\bar{A}_{11}, \bar{B}_{11}, \bar{D}_{11}\right]=\int_{A} E(z)\left[1, z, z^{2}\right] d A
$$

where $E(z)$ is the effective elasticity modulus calculated by Eq. (2.4).

The shear stiffness coefficient is given as follows, where $k$ is the shear correction factor and $G(z)$ is the effective shear modulus, found from Eq. (2.4)

$$
\bar{A}_{55}=k \int_{A} G(z) d A
$$

The kinetic energy expression is the same for both types of beams, and is given by

$$
T=\frac{1}{2} \int_{0}^{L}\left[I_{1}\left(\dot{u}_{0}\right)^{2}+I_{1}(\dot{w})^{2}+2 I_{2} \dot{u}_{0} \dot{\theta}+I_{3}(\dot{\theta})^{2}\right] d x
$$

where $I_{1}, I_{2}$ and $I_{3}$ are the inertial characteristics of the beam, and are given below

$$
\left[I_{1}, I_{2}, I_{3}\right]=\int_{A} \rho(z)\left[1, z, z^{2}\right] d A
$$

where $\rho(z)$ is the effective material density, calculated by Eq. (2.4). 
The centrifugal force in Eq. $(3.1)_{1}$ is

$$
F_{C F}=\int_{0}^{L} \rho A \Omega^{2}(R+x) d x=\int_{0}^{L} I_{1} \Omega^{2}(R+x) d x
$$

\section{Finite element modeling}

Finite element formulation of the rotating FG beam that undergoes axial and transverse displacement is carried out in this Section. The global finite element model of the beam used for the formulation is illustrated in Fig. 4a. In the case of the rotating beam, additional terms appear in the element matrices due to the centrifugal force. Finite element representation of the centrifugal force, given in Fig. 4b, can be used.

(a)

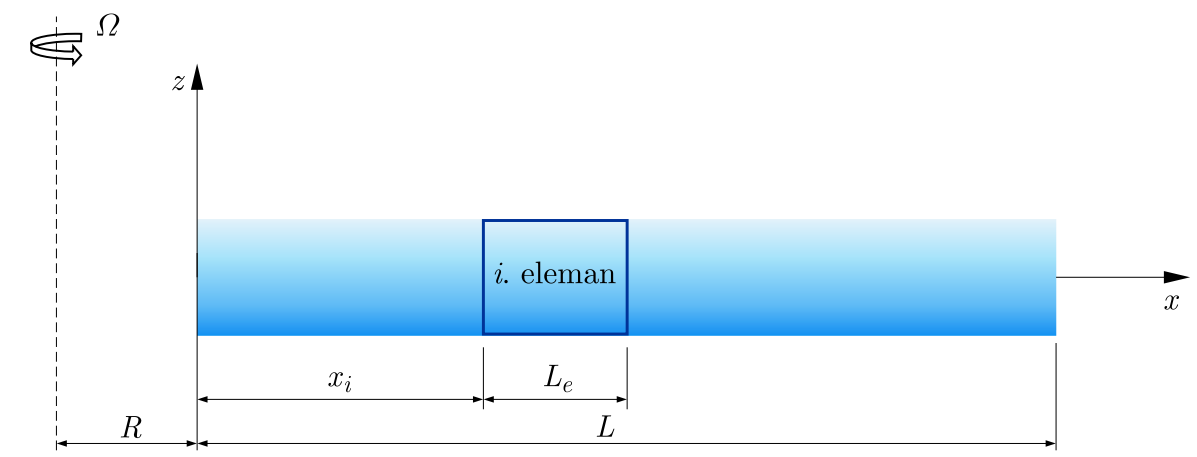

(b)

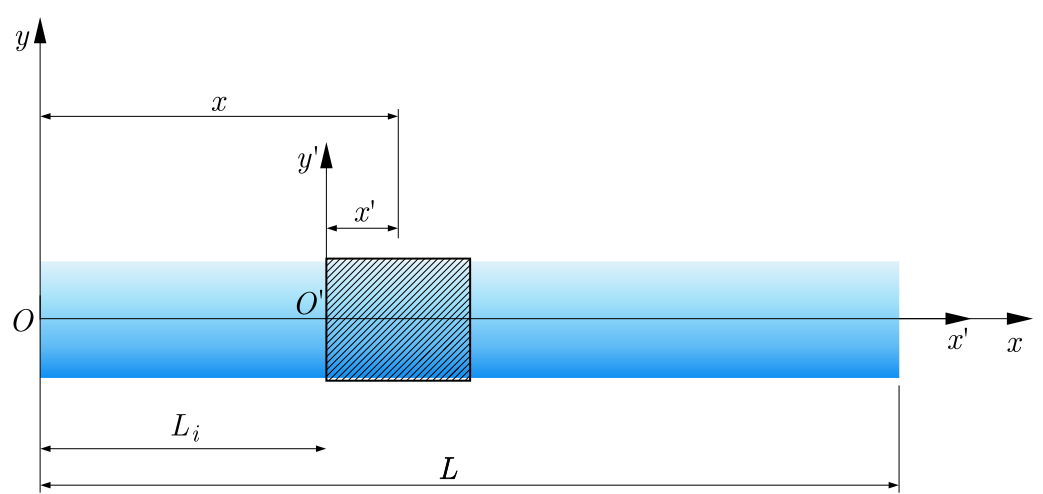

Fig. 4. Finite element model of: (a) rotating FG beam, (b) centrifugal force

Here, $L_{i}$ is the offset of each element from the rotational axis, $x y z$ is the global coordinate system while $x^{\prime} y^{\prime} z^{\prime}$ is the local coordinate system.

Referring to Fig. 4b, the centrifugal force given by Eq. (3.6) can be expressed in the finite element form as follows

$$
F_{C F}(x)=I_{1} \Omega^{2}\left[R\left(L-L_{i}-x^{\prime}\right)+\frac{1}{2}\left(L-L_{i}-x^{\prime}\right)\left(L+L_{i}+x^{\prime}\right)\right]
$$

where the offset of each element from the rotational axis is given by

$$
L_{i}=(i-1) \frac{L}{N_{e}}
$$

Here, $L$ is length of the whole beam and $N_{e}$ is the number of elements the beam is divided. 


\subsection{Finite element modeling of the Euler-Bernoulli beam}

The finite element model of the rotating Euler-Bernoulli beam element that undergoes axial displacement $u$ and transverse displacement $w$ is given in Fig. 5 .

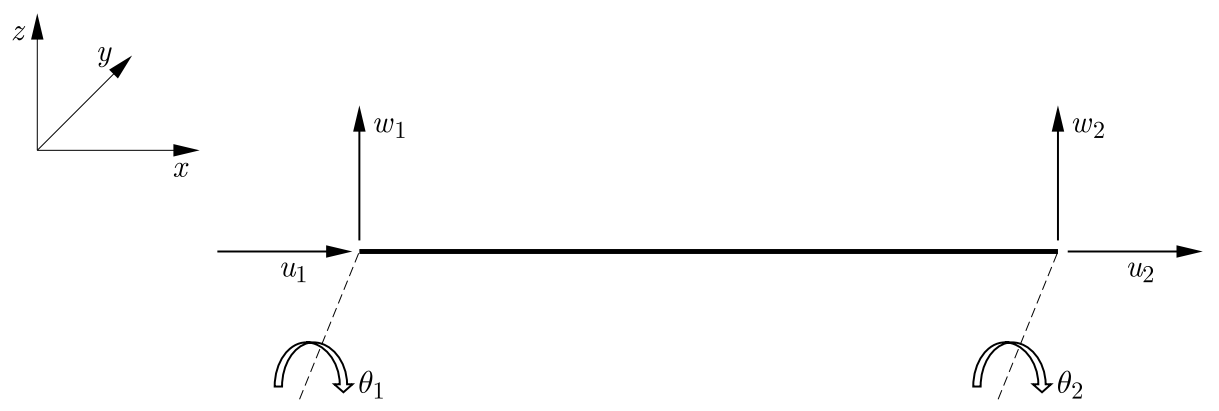

Fig. 5. Finite element model of the Euler-Bernoulli beam element

Here, a two noded beam element that has six degrees of freedom is preferred for the modelling. Here, $u$ is the axial displacement, $w$ is the transverse displacement and $\theta$ is the angle due to transverse displacement.

Polynomials of appropriate order are defined for the displacement field as follows (Hartmann and Katz, 2004)

$$
\begin{aligned}
& u=a_{0}+a_{1} x \quad w=a_{2}+a_{3} x+a_{4} x^{2}+a_{5} x^{3} \\
& \theta=w^{\prime}=a_{3}+2 a_{4} x+3 a_{5} x^{2}
\end{aligned}
$$

Considering the displacement field polynomials given by Eq. (4.3), the nodal displacements are determined as displacement values at the first node of the element $x=0$ and at the second node $x=L_{e}$, respectively. These are given in a matrix form as follows

$$
\left\{\begin{array}{c}
u_{1} \\
w_{1} \\
\theta_{1} \\
u_{2} \\
w_{2} \\
\theta_{2}
\end{array}\right\}=\left[\begin{array}{cccccc}
1 & 0 & 0 & 0 & 0 & 0 \\
0 & 0 & 1 & 0 & 0 & 0 \\
0 & 0 & 0 & 1 & 0 & 0 \\
1 & L_{e} & 0 & 0 & 0 & 0 \\
0 & 0 & 1 & L_{e} & L_{e}^{2} & L_{e}^{3} \\
0 & 0 & 0 & 1 & 2 L_{e} & 3 L_{e}^{2}
\end{array}\right]\left\{\begin{array}{l}
a_{0} \\
a_{1} \\
a_{2} \\
a_{3} \\
a_{4} \\
a_{5}
\end{array}\right\}
$$

Here, $(\cdot)_{1}$ are displacement values of the 1 st node while $(\cdot)_{2}$ are displacements on the 2 nd node.

The relation between the displacement field and nodal displacements is

$$
\mathbf{q}=\mathbf{N q}_{e}
$$

where, in the present beam model, the expressions of displacements $\mathbf{q}$, nodal displacements $\mathbf{q}_{e}$ and the matrix of shape functions $\mathbf{N}$ are given by

$$
\mathbf{q}=[u, w, \theta]^{\mathrm{T}} \quad \mathbf{N}=\left[\mathbf{N}_{u}, \mathbf{N}_{w}, \mathbf{N}_{\theta}\right]^{\mathrm{T}} \quad \mathbf{q}_{e}=\left[u_{1}, w_{1}, \theta_{1}, u_{2}, w_{2}, \theta_{2}\right]^{\mathrm{T}}
$$

where the expressions of the shape functions are

$$
\begin{aligned}
& \mathbf{N}_{u}=\left[1-\frac{x}{L}, 0,0, \frac{x}{L}, 0,0\right] \\
& \mathbf{N}_{w}=\left[0,1-\frac{3 x^{2}}{L_{e}^{2}}+\frac{2 x^{3}}{L_{e}^{3}}, x-\frac{2 x^{2}}{L_{e}}+\frac{x^{3}}{L_{e}^{2}}, 0, \frac{3 x^{2}}{L_{e}^{2}}-\frac{2 x^{3}}{L_{e}^{3}},-\frac{x^{2}}{L_{e}}+\frac{x^{3}}{L_{e}^{2}}\right] \\
& \mathbf{N}_{\theta}=\left[0,-\frac{6 x}{L_{e}^{2}}+\frac{6 x^{2}}{L_{e}^{3}}, 1-\frac{4 x}{L_{e}}+\frac{3 x^{2}}{L_{e}^{2}}, 0, \frac{6 x}{L_{e}^{2}}-\frac{6 x^{2}}{L_{e}^{3}},-\frac{2 x}{L_{e}}+\frac{3 x^{2}}{L_{e}^{2}}\right]
\end{aligned}
$$


Here, $\mathbf{N}_{u}, \mathbf{N}_{w}$ and $\mathbf{N}_{\theta}$ are the shape functions associated with the axial displacement $u$, transverse displacement $w$ and the angle due to transverse displacement $\theta$, respectively.

Considering the effect of the centrifugal force and substituting the shape functions, i.e. Eqs. $(4.7)_{1,2}$, into the potential and kinetic energy expressions, i.e. Eq. (3.1) $)_{1}$ and Eq. (3.4), the element stiffness matrix $\mathbf{K}^{e}$ and element mass matrix $\mathbf{M}^{e}$ are obtained as follows

$$
\begin{aligned}
& \mathbf{K}^{e}=\frac{1}{2} \int_{0}^{L_{e}}\left(\bar{A}_{11}\left[\frac{d \mathbf{N}_{u}}{d x}\right]^{\mathrm{T}} \frac{d \mathbf{N}_{u}}{d x}+2 \bar{B}_{11}\left[\frac{d \mathbf{N}_{u}}{d x}\right]^{\mathrm{T}} \frac{d \mathbf{N}_{\theta}}{d x}\left[\frac{d \mathbf{N}_{\theta}}{d x}\right]^{\mathrm{T}} \frac{d \mathbf{N}_{u}}{d x}+\bar{D}_{11}\left[\frac{d \mathbf{N}_{\theta}}{d x}\right]^{\mathrm{T}} \frac{d \mathbf{N}_{\theta}}{d x}\right) d x \\
& \mathbf{M}^{e}=\frac{1}{2} \int_{0}^{L_{e}}\left(I_{1} \mathbf{N}_{u}^{\mathrm{T}} \mathbf{N}_{u}+I_{1} \mathbf{N}_{w}^{\mathrm{T}} \mathbf{N}_{w}+2 I_{2} \mathbf{N}_{u}^{\mathrm{T}} \mathbf{N}_{u} \mathbf{N}_{\theta}^{\mathrm{T}} \mathbf{N}_{\theta}+I_{3} \mathbf{N}_{\theta}^{\mathrm{T}} \mathbf{N}_{\theta}\right) d x
\end{aligned}
$$

The element stiffness matrix is derived from the potential energy expression, and the element mass matrice is derived from the kinetic energy expression.

\subsection{Finite element modeling of the Timoshenko beam}

The finite element model of the rotating Timoshenko beam element that undergoes axial displacement $u$ and transverse displacement $w$ is given in Fig. 6. Here, it is seen that the two noded beam element having eight degrees of freedom is preferred to model the beam. Here, $\theta$ is the angle due to flapwise bending and $\varphi$ is the shear angle which results from the Timoshenko beam formulation.

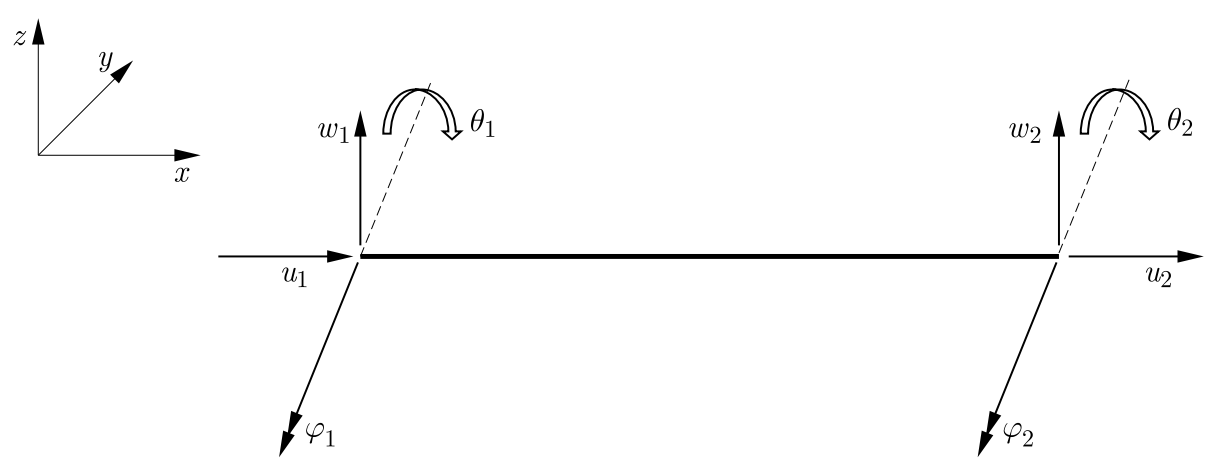

Fig. 6. Finite element model of the Timoshenko beam element

Polynomials of appropriate order are defined for the displacement field as follows (Hartmann and Katz, 2004)

$$
\begin{array}{ll}
u=a_{0}+a_{1} x & w=a_{2}+a_{3} x+a_{4} x^{2}+a_{5} x^{3} \\
\varphi=a_{6}+a_{7} x & \theta=w^{\prime}-\varphi=a_{3}-a_{6}+\left(2 a_{4}-a_{7}\right) x+3 a_{5} x^{2}
\end{array}
$$

As described in the finite element modeling of the Euler-Bernoulli beam, the shape functions are obtained as follows

$$
\begin{aligned}
& \mathbf{N}_{u}=\left[1-\frac{x}{L}, 0,0,0, \frac{x}{L}, 0,0,0\right] \\
& \mathbf{N}_{w}=\left[0,1-\frac{3 x^{2}}{L_{e}^{2}}+\frac{2 x^{3}}{L_{e}^{3}}, x-\frac{2 x^{2}}{L_{e}}+\frac{x^{3}}{L_{e}^{2}}, x-\frac{2 x^{2}}{L_{e}}+\frac{x^{3}}{L_{e}^{2}}, 0, \frac{3 x^{2}}{L_{e}^{2}}-\frac{2 x^{3}}{L_{e}^{3}},-\frac{x^{2}}{L_{e}}+\frac{x^{3}}{L_{e}^{2}},-\frac{x^{2}}{L_{e}}+\frac{x^{3}}{L_{e}^{2}}\right] \\
& \mathbf{N}_{\theta}=\left[0,-\frac{6 x}{L_{e}^{2}}+\frac{6 x^{2}}{L_{e}^{3}}, 1-\frac{4 x}{L_{e}}+\frac{3 x^{2}}{L_{e}^{2}},-\frac{3 x}{L_{e}}+\frac{3 x^{2}}{L_{e}^{2}}, 0, \frac{6 x}{L_{e}^{2}}-\frac{6 x^{2}}{L_{e}^{3}},-\frac{2 x}{L_{e}}+\frac{3 x^{2}}{L_{e}^{2}},-\frac{3 x}{L_{e}}+\frac{3 x^{2}}{L_{e}^{2}}\right] \\
& \mathbf{N}_{\varphi}=\left[0,0,0,1-\frac{x}{L}, 0,0,0, \frac{x}{L}\right]
\end{aligned}
$$


Here, $\mathbf{N}_{u}, \mathbf{N}_{w}, \mathbf{N}_{\theta}$ and $\mathbf{N}_{\varphi}$ are the shape functions associated with the axial displacement $u$, transverse displacement $w$, angle due to transverse displacement $\theta$ and the shear angle $\varphi$, respectively.

\subsection{Stiffness and mass matrices}

Considering the effect of the centrifugal force and substituting the shape functions, i.e. Eqs. (4.10) into the potential and kinetic energy expressions Eq. (3.1) 2 and (3.4), the element stiffness matrix $\mathbf{K}^{e}$ and element mass matrix $\mathbf{M}^{e}$ are obtained as follows

$$
\begin{aligned}
\mathbf{K}^{e} & =\frac{1}{2} \int_{0}^{L_{e}}\left(\bar{A}_{11}\left[\frac{d \mathbf{N}_{u}}{d x}\right]^{\mathrm{T}} \frac{d \mathbf{N}_{u}}{d x}+2 \bar{B}_{11}\left[\frac{d \mathbf{N}_{u}}{d x}\right]^{\mathrm{T}} \frac{d \mathbf{N}_{\theta}}{d x}\left[\frac{d \mathbf{N}_{\theta}}{d x}\right]^{\mathrm{T}} \frac{d \mathbf{N}_{u}}{d x}\right. \\
& \left.+\bar{D}_{11}\left[\frac{d \mathbf{N}_{\theta}}{d x}\right]^{\mathrm{T}} \frac{d \mathbf{N}_{\theta}}{d x}+\bar{A}_{55}\left(\frac{d \mathbf{N}_{w}}{d x}-\mathbf{N}_{\theta}\right)^{\mathrm{T}}\left(\frac{d \mathbf{N}_{w}}{d x}-\mathbf{N}_{\theta}\right)\right) d x \\
\mathbf{M}^{e} & =\frac{1}{2} \int_{0}^{L_{e}}\left(I_{1} \mathbf{N}_{u}^{\mathrm{T}} \mathbf{N}_{u}+I_{1} \mathbf{N}_{w}^{\mathrm{T}} \mathbf{N}_{w}+2 I_{2} \mathbf{N}_{u}^{\mathrm{T}} \mathbf{N}_{u} \mathbf{N}_{\theta}^{\mathrm{T}} \mathbf{N}_{\theta}+I_{3} \mathbf{N}_{\theta}^{\mathrm{T}} \mathbf{N}_{\theta}\right) d x
\end{aligned}
$$

\subsection{Global matrices and modal analysis}

Depending on the number of elements used in the finite element modeling, all the element matrices are assembled by considering the finite element rules to obtain the global matrices. The boundary conditions given in Table 1 for different cases are applied to the global matrices to get reduced matrices, and the following matrix system of equations are obtained

$$
\mathbf{M} \ddot{\mathbf{q}}+\mathbf{K q}=\mathbf{0}
$$

where $\mathbf{M}$ and $\mathbf{K}$ is the reduced global mass and global stiffness matrix, respectively. The eliminated degrees of freedom are given in Table 1.

Table 1. Eliminated degrees of freedom in FEM for different boundary conditions

\begin{tabular}{|c|c|c|c|c|}
\hline \multirow{2}{*}{$\begin{array}{c}\text { Boundary } \\
\text { conditions }\end{array}$} & \multicolumn{2}{|c|}{ Euler beam FEM } & \multicolumn{3}{c|}{ Timoshenko beam FEM } \\
\cline { 2 - 5 } & $x=0$ & $x=L_{e}$ & $x=0$ & $x=L_{e}$ \\
\hline \hline Clamped-free & $u=w=\theta=0$ & - & $u=w=\theta=\varphi=0$ & - \\
\hline Simple-simple & $u=w=0$ & $u=w=0$ & $u=w=0$ & $u=w=0$ \\
\hline
\end{tabular}

Modal analysis is applied to Eq. (4.12) to calculate the natural frequencies. Solving the following determinant, the natural frequencies are calculated for the Euler-Benoulli and Timoshenko beam models

$$
\operatorname{det}\left(\mathbf{K}-\omega^{2} \mathbf{M}\right)=0
$$

where $\omega$ represents the natural frequencies of the rotating system. In the following Sections, the natural frequencies are declared in a nondimensional form $\lambda$, i.e. Eq. (5.1).

\section{Results and discussions}

In this Section, effects of several parameters, i.e. material distribution, rotational speed, slenderness ratio and the power law index on vibration characteristics are examined for both Euler-Bernoulli and Timoshenko beams having different boundary conditions and material distribution properties. 


\subsection{Dimensionless parameters}

To make comparisons with the studies in open literature, the following dimensionless parameters are defined (Özdemir, 2016)

$$
\begin{array}{ccc}
\bar{x}=\frac{x}{L} & \bar{\Omega}^{2}=\frac{I_{1} L^{4} \Omega^{2}}{\bar{D}_{11}} & \lambda=\frac{\omega L^{2}}{h} \sqrt{\frac{\rho}{E}} \\
r^{2}=\frac{I_{3}}{I_{1} L^{2}} & \bar{w}=\frac{w}{L} & \bar{u}_{0}=\frac{u_{0}}{L}
\end{array}
$$

Here, $\lambda$ is the dimensionless frequency parameter, $\bar{\Omega}$ is the dimensionless angular speed parameter and $r$ is the inverse of the slenderness ratio parameter.

\section{Results for functionally graded beams}

In this Section, vibration characterics of FG Euler-Bernoulli and Timoshenko beams are examined for two different material distributions.

In the first case, the FG beam is made of Aluminum (Al) at the top and Alumina $\left(\mathrm{Al}_{2} \mathrm{O}_{3}\right)$ at the bottom. The effective beam properties change through the beam thickness according to the power law, Eq. (2.4). The material properties of the FG beam are displayed in Table 2 for the 1st case.

Table 2. Material properties of functionally graded beam model (1st case)

\begin{tabular}{|l|c|c|}
\hline \multicolumn{1}{|c|}{ Material property } & Aluminum $(\mathrm{Al})$ & Alumina $\left(\mathrm{Al}_{2} \mathrm{O}_{3}\right)$ \\
\hline \hline Modulus of elasticity $E$ & $70 \mathrm{GPa}$ & $380 \mathrm{GPa}$ \\
\hline Density $\rho$ & $2702 \mathrm{~kg} / \mathrm{m}^{3}$ & $3960 \mathrm{~kg} / \mathrm{m}^{3}$ \\
\hline Poisson's ratio $\nu$ & 0.3 & 0.3 \\
\hline
\end{tabular}

\begin{tabular}{|c|c|c|c|c|c|c|c|c|c|}
\hline BC's & $L / h$ & $\lambda=\frac{\omega L^{2}}{h} \sqrt{\frac{\rho_{m}}{E_{m}}}$ & $n=0$ & $n=0.2$ & $n=0.5$ & $n=1$ & $n=2$ & $n=5$ & $n=10$ \\
\hline \multirow{6}{*}{ C. } & \multirow{3}{*}{5} & Present & 3846 & 1.80388 & 1.65105 & 1.49267 & 1.36168 & 1.29559 & .25751 \\
\hline & & Lee and Lee (2017) & 1.9385 & 1.80 & 1.6504 & 1.4914 & 1.3599 & 1.2942 & 1.2565 \\
\hline & & Şimşek (2010) & $\overline{1.93}$ & 1.80 & 57 & 1.49 & $1.35 !$ & 1.29 & 1.25648 \\
\hline & & Present & 1.95249 & 1.81 & 1.663 & 1.50425 & 1.37325 & 1.30728 & 1.26816 \\
\hline & & Lee and Lee (2017) & 1.95 & 1.8 & & 1.5 & 1.3 & 1.3057 & 1.2671 \\
\hline & & Şimşek (2010) & 1.95248 & & & 1.50293 & 1.37142 & 1.30574 & 1.26 \\
\hline \multirow{6}{*}{ SS } & \multirow{3}{*}{5} & Present & 12.1826 & 11.3371 & 10.3714 & 9.36547 & 8.52944 & 8.11103 & 7.88057 \\
\hline & & Lee and Lee (2017) & 12.1826 & 11.3369 & 10.3 & 9.3642 & 8.5277 & 8.1096 & 7.8797 \\
\hline & & Şimşek (2010) & 12.1826 & 11.3398 & 10.3 & 9.36422 & 8.52772 & 8.10955 & 7.87968 \\
\hline & \multirow{3}{*}{20} & Present & 12.4143 & 509 & & 9.55664 & 8.72028 & 8.30209 & 8.05 \\
\hline & & Lee and Lee (2017) & 12.4143 & & & & 8.7186 & 8.3006 & 8.0556 \\
\hline & & Şimşek (2010) & 12.4142 & 11.5537 & 10.5713 & 9.55538 & 8.71856 & 8.30064 & 8.05560 \\
\hline
\end{tabular}

Table 3. The natural frequencies of nonrotating FG Euler-Bernoulli beam (CF and SS ends)

Variation of the fundamental frequencies of the FG nonrotating Euler-Bernoulli beam with respect to the slenderness ratio and power law index parameter are given in Table 3 for different boundary conditions, i.e. clamped-free (CF) and simply-simply supported (SS). The calculated results are compared with those given by Şimşek (2010), where the Lagrange multipliers method was used, and those given by Lee and Lee (2017), where Transfer Matrix Method was applied. 
The convergence of the Euler-Bernoulli beam frequencies given in Table 3 is drawn in Fig. 7 . When finite element runs are analysed, it is noticed that the fundamental natural frequencies of the Euler-Bernoulli beam having the slenderness ratio of $L / h=20$ get converged with $N=260$ elements for $n=10$ and with $N=320$ elements for $n=5$. At the same time fundamental natural frequencies of the Euler beam having the slenderness ratio of $L / h=5$ get converged with $N=180$ elements for $n=10$ and with $N=240$ elements for $n=5$. Consequently, as the power law index parameter increases, and as the slenderness ratio decreases, the fundamental frequencies converge more rapidly.

(a)

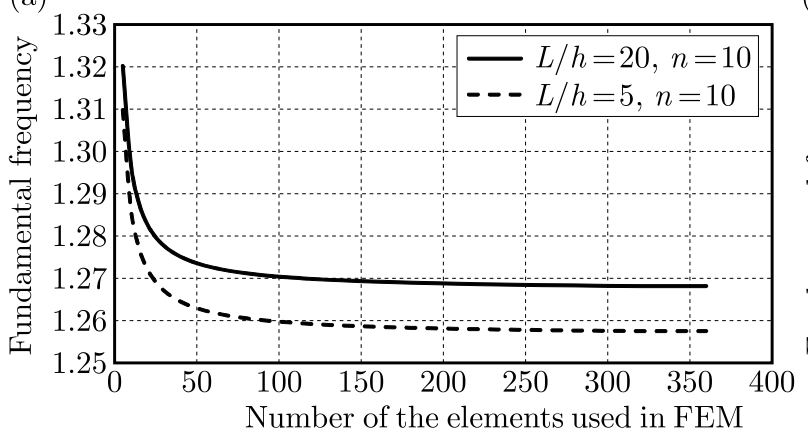

(b)

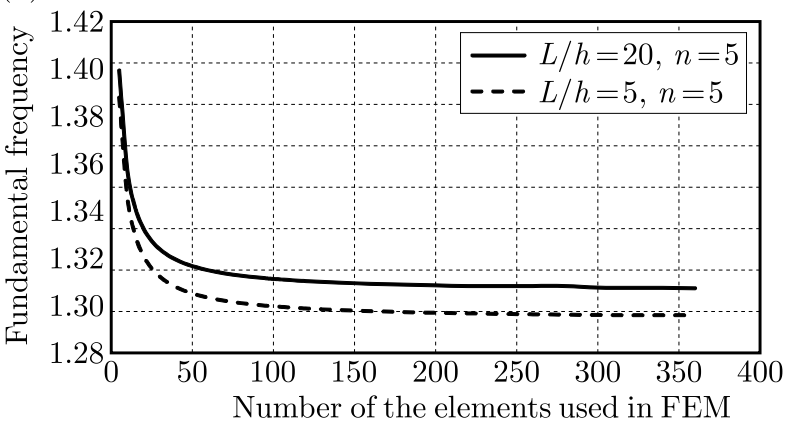

Fig. 7. Convergence rate of the natural frequencies with respect to the slenderness ratio and the power law index parameter

Variation of the fundamental frequencies of the FG nonrotating Timoshenko beam of the slenderness ratio of $L / h=5$ with respect to the power law index parameter is given in Table 4 for different boundary conditions, i.e. clamped-free (CF) and simply-simply supported (SS) beams. The calculated results are compared with those given by Şimşek (2010), where the Lagrange multipliers method is used and with those given by Nguyen et al. (2015), where a new high order shear deformation theory is applied.

Table 4. The natural frequencies of a nonrotating FG Timoshenko beam $(L / h=5)$

\begin{tabular}{|c|c|c|c|c|c|}
\hline \multirow{2}{*}{ BC's } & $\lambda=\frac{\omega L^{2}}{h} \sqrt{\frac{\rho_{m}}{E_{m}}}$ & $n=0$ & $n=0.5$ & $n=1$ & $n=2$ \\
\hline \hline \multirow{3}{*}{$\mathrm{CF}$} & Present & 1.89482 & 1.61724 & 1.46304 & 1.33380 \\
\cline { 2 - 6 } & Şimşek $(2010)$ & 1.89523 & 1.61817 & 1.46328 & 1.33254 \\
\cline { 2 - 6 } & Nguyen et al. $(2015)$ & 1.8957 & 1.6182 & 1.4636 & 1.3328 \\
\hline \multirow{2}{*}{ SS } & Present & 10.015 & 8.68448 & 7.91205 & 7.19908 \\
\cline { 2 - 6 } & Şimşek (2010) & 10.0705 & 8.74674 & 7.95034 & 7.17674 \\
\cline { 2 - 6 } & Nguyen et al. $(2015)$ & 10.0726 & 8.7463 & 7.9518 & 7.1776 \\
\hline
\end{tabular}

When Tables 3 and 4 are examined, it is noticed that the increasing power law index parameter has a decreasing effect on the natural frequencies. Moreover, the slenderness ratio has a small increasing effect on the natural frequencies while the frequencies of clamped-free beams are higher than the frequencies of simply-simply supported beams.

Variation of the fundamental frequencies of the FG rotating Timoshenko beam with respect to the power law index parameter and slenderness ratio is given in Table 5 where the dimensionless angular velocity is $\bar{\Omega}=5$. The calculated results are compared with those given by Özdemir (2019), where the differential transform method (DTM) which is an analytical method that gives accurate results is applied.

When Table 5 is examined, it is noticed that there is a slight difference between the present results and calculated by DTM because FEM is a numerical method while DTM is an analytical method. 
Table 5. Natural frequencies of the rotating FG Timoshenko beam with CF ends

\begin{tabular}{|c|c|c|c|c|c|c|c|c|}
\hline \multirow{2}{*}{$L / h$} & \multicolumn{2}{|c|}{$n=0$} & \multicolumn{2}{c|}{$n=0.2$} & \multicolumn{2}{c|}{$n=0.5$} & \multicolumn{2}{c|}{$n=5$} \\
\cline { 2 - 9 } & $\begin{array}{c}\text { Ozdemir } \\
(2019)\end{array}$ & Present & $\begin{array}{c}\text { Ozdemir } \\
(2019)\end{array}$ & Present & $\begin{array}{c}\text { Ozdemir } \\
(2019)\end{array}$ & Present & $\begin{array}{c}\text { Ozdemir } \\
(2019)\end{array}$ & Present \\
\hline \hline 4 & 3.4358 & 3.4569 & 2.8159 & 2.9013 & 2.6592 & 2.7753 & 2.4888 & 2.5877 \\
\hline 5 & 3.4834 & 3.4976 & 2.8536 & 2.9351 & 2.6968 & 2.8099 & 2.5273 & 2.6227 \\
\hline
\end{tabular}

In Fig. 8, variation of the natural frequencies of Euler-Bernoulli and Timoshenko beams with respect to the power law index parameter $n$ is demonstrated for the slenderness ratio $L / h=10$. The Euler-Bernoulli frequencies are shown with solid lines while the Timoshenko frequencies are shown by dashed lines. When Fig. 8 is examined, it is noticed that the decreasing effect of the power law index parameter is more dominant on higher frequencies. Additionally, the difference between the Euler and Timoshenko theories gets more obvious in higher modes, as expected.

(a)

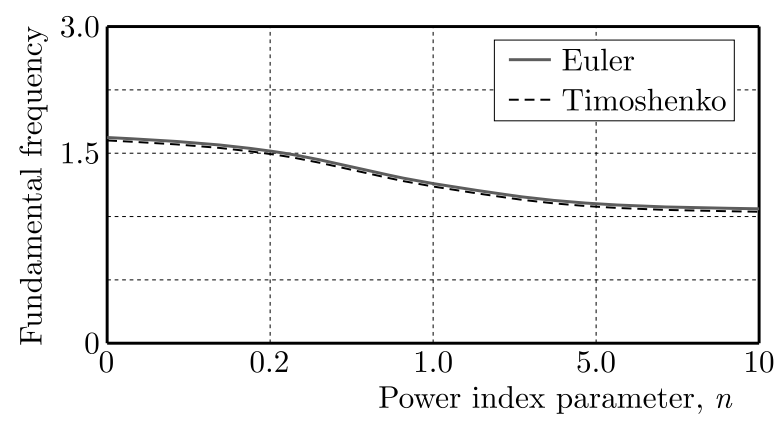

(b)

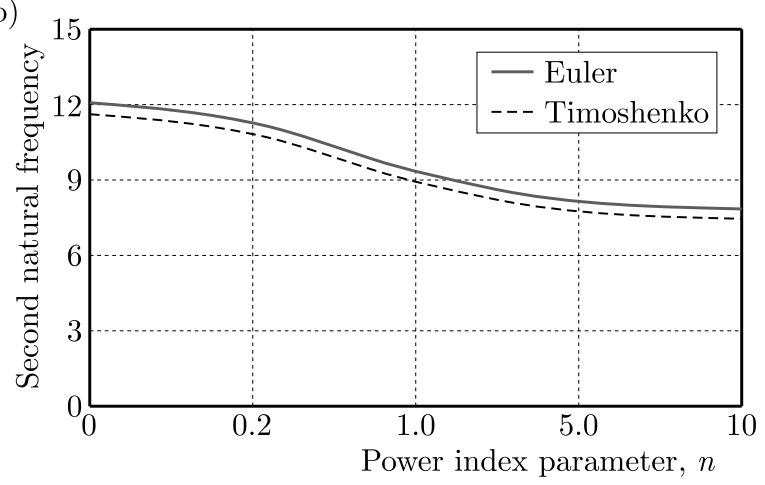

(c)

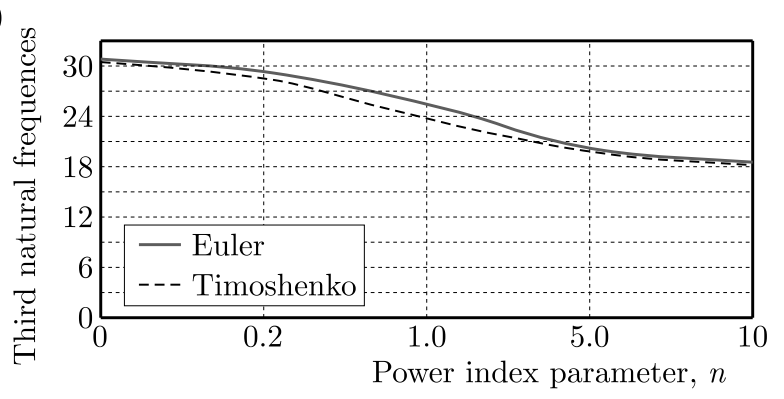

Fig. 8. Variation of Euler-Bernoulli and Timoshenko beam frequencies with respect to the power law index parameter

As the 2nd case, the rotating functionally graded Timoshenko beam with clamped free boundary conditions is analyzed and the material properties of this beam model is given in Table 6 . The beam is made of steel and alumina, and its geometrical properties are such that $h=22.13 \mathrm{~mm}, b=2.66 \mathrm{~mm}, L=152.40 \mathrm{~mm}$.

Table 6. Material properties of the functionally graded rotating Timoshenko beam model (2nd case)

\begin{tabular}{|l|c|c|}
\hline \multicolumn{1}{|c|}{ Material property } & Steel (metal) & Alumina (ceramic) \\
\hline \hline Modulus of elasticity $E$ & $214 \mathrm{GPa}$ & $390 \mathrm{GPa}$ \\
\hline Modulus of rigidity $G$ & $82.2 \mathrm{GPa}$ & $137 \mathrm{GPa}$ \\
\hline Density $\rho$ & $2800 \mathrm{~kg} / \mathrm{m}^{3}$ & $3200 \mathrm{~kg} / \mathrm{m}^{3}$ \\
\hline Poisson's ratio $\nu$ & 0.3 & 0.34 \\
\hline
\end{tabular}


Variation of the first three natural frequencies of the rotating functionally graded Timoshenko beam with respect to the power index parameter $n$ and the dimensionless rotational speed $\bar{\Omega}$ is tabulated in Table 7 . When Table 7 is analyzed, as expected, it is noticed that the rotational speed has an increasing effect on the natural frequencies due to the stiffening effect of the centrifugal force.

Table 7. Variation of the natural frequencies of the rotating FG Timoshenko beam with respect to $n$ and $\Omega$

\begin{tabular}{|c|c|c|c|c|c|}
\hline \multirow{\Omega}{*}{$\Omega$} & \multicolumn{5}{|c|}{ Power law exponent $n$} \\
\cline { 2 - 6 } & 0 & 0.2 & 0.5 & 1 & 2 \\
\hline \hline \multirow{4}{*}{0} & 2.1044 & 1.8009 & 1.5788 & 1.4125 & 1.2837 \\
\cline { 2 - 6 } & 12.0822 & 10.3425 & 9.0692 & 8.1098 & 7.3583 \\
\cline { 2 - 6 } & 30.3353 & 25.9775 & 22.7841 & 20.3616 & 18.4423 \\
\cline { 2 - 6 } & 52.6304 & 45.0897 & 39.5540 & 35.3266 & 31.9426 \\
\hline \multirow{4}{*}{2} & 2.4810 & 2.1231 & 1.8613 & 1.6653 & 1.5136 \\
\cline { 2 - 6 } & 12.4466 & 10.6536 & 9.3419 & 8.3544 & 7.5816 \\
\cline { 2 - 6 } & 30.7289 & 26.3125 & 23.0778 & 20.6258 & 18.6848 \\
\cline { 2 - 6 } & 53.0853 & 45.4758 & 39.8925 & 35.6319 & 32.2243 \\
\hline \multirow{4}{*}{5} & 3.8725 & 3.3138 & 2.9051 & 2.5993 & 2.3626 \\
\cline { 2 - 6 } & 14.2034 & 12.1537 & 10.6571 & 9.5336 & 8.6574 \\
\cline { 2 - 6 } & 32.7030 & 27.9932 & 24.5513 & 21.9509 & 19.9004 \\
\cline { 2 - 6 } & 55.3945 & 47.4366 & 41.6114 & 37.1819 & 33.6533 \\
\hline
\end{tabular}

\section{Conclusion}

In this study, finite element formulation of functionally graded Euler-Bernoulli and Timoshenko beams that undergo axial and transverse displacements is derived step by step. The calculated results are presented in several figures and tables and compared with those from open literature.

Considering the calculated results, the following conclusions are reached:

- As the slenderness ratio $L / h$ increases, the natural frequencies increase.

- The natural frequencies decrease as the value of the power law exponent $n$ increases, and the decreasing effect of the power law index parameter is more dominant on higher frequencies.

- As the power law index parameter increases and as the slenderness ratio decreases, the fundamental frequencies converge more rapidly.

- The rotational speed has an increasing effect on the natural frequencies.

\section{References}

1. Alshorbagy A.E., Eltaher M.A., Mahmoud F.F., 2011, Free vibration characteristics of a functionally graded beam by finite element method, Applied Mathematical Modelling, 35, 412-425

2. Giunta G., Crisafulli D., Belouettar S., Carrera E., 2011, Hierarchical theories for the free vibration analysis of functionally graded beams, Composite Structures, 94, 68-74

3. Han S.M., Benaroya H., Wei T., 1999, Dynamics of transversely vibrating beams using four engineering theories, Journal of Sound and Vibration, 225, 5, 935-988

4. Hartmann F., Katz C., 2004, Structural Analysis with Finite Elements, Springer

5. HuANG Y., Li X.F., 2010, A new approach for free vibration of axially functionally graded beams with non-uniform cross-section, Journal of Sound and Vibration, 329, 2291-2303 
6. Lai S.K., Harrington J., Xiang Y., Chow K.W., 2012, Accurate analytical perturbation approach for large amplitude vibration of functionally graded beams, International Journal of Non-Linear Mechanics, 47, 473-480

7. Latalski J., Warminski J., Rega G., 2017, Bending-twisting vibrations of a rotating hub-thin-walled composite beam system, Mathematics and Mechanics of Solids, 22, 6

8. LEE J.W., LEE J.Y., 2017, Free vibration analysis of functionally graded Bernoulli-Euler beams using an exact transfer matrix expression, International Journal of Mechanical Sciences, 122, 1-17

9. Loja M.A.R., Barbosa J.I., Mota Soares C.M., 2012, A study on the modelling of sandwich functionally graded particulate composite, Composite Structures, 94, 2209-2217

10. Loy C.T., LAm K.Y., Reddy J.N., 1999, Vibration of functionally graded cylindrical shells, International Journal of Mechanical Science, 41, 309-324

11. Nguyen T.K., Nguyen T.T.P., Vo T.P., Thai H.T., 2015, Vibration and buckling analysis of functionally graded sandwich beams by a new higher-order shear deformation theory, Composites, Part B: Engineering, 76, 273-285

12. ÖzDEmir Ö., 2019, Vibration analysis of rotating Timoshenko beams with different material distribution properties, Selcuk University, Journal of Science, Engineering and Technology, 7, 2, 272-286

13. ŞıMŞEK M., 2010, Fundamental frequency analysis of functionally graded beams by using different higher-order beam theories, Nuclear Engineering and Design, 240, 4, 697-705

14. Thai H.T., Vo T.P., 2012, Bending and free vibration of functionally graded beams using various higher-order shear deformation beam theories, International Journal of Mechanical Sciences, 62, $57-66$

15. Wattanasakulpong N., Prusty B.G., Kelly D.W., Hoffman M., 2012, Free vibration analysis of layered functionally graded beams with experimental validation, Materials and Design, 36, 182-190 